\title{
Halophenol Rearrangement in Lewis Acid-Catalyzed Friedel-Crafts Conditions: Evidence of Competitive Initial Protonation and Acylation
}

\begin{abstract}
Halogen rearrangement was observed during the Lewis acid-catalyzed Friedel-Crafts reaction of phthalic anhydride with bromophenols or bromoanisole. Further investigation revealed that 2-, 3-, and 4-bromophenols undergo rearrangement into other isomers under these reaction conditions. Product distribution from these reactions suggested that halogen rearrangement takes place during the s-complex intermediate of the condensation step. Furthermore, iodophenol undergoes hydrodeiodination rapidly rather than rearrangement, whereas chlorophenol does not undergo rearrangement at all.
\end{abstract}

